\title{
Systoles and kissing numbers of finite area hyperbolic surfaces
}

\author{
FEDERICA FANONI \\ Hugo PARLIER
}

\begin{abstract}
We study the number and the length of systoles on complete finite area orientable hyperbolic surfaces. In particular, we prove upper bounds on the number of systoles that a surface can have (the so-called kissing number for hyperbolic surfaces). Our main result is a bound which only depends on the topology of the surface and which grows subquadratically in the genus.
\end{abstract}

$30 \mathrm{~F} 10 ; 32 \mathrm{G} 15,53 \mathrm{C} 22$

\section{Introduction}

In analogy with classical sphere packing problems in $\mathbb{R}^{n}$, Schmutz Schaller named and studied kissing numbers for hyperbolic surfaces. This is a particular instance of a more general analogy between the study of $n$-dimensional lattices (and their parameter spaces) and the study of hyperbolic surfaces (and their parameter spaces). Both are natural generalizations of the study of 2-dimensional flat tori. The natural parameter spaces of these tori are $\mathbb{H}$ and $\mathbb{H} / \mathrm{PSL}_{2}(\mathbb{Z})$; their higher-dimensional analogues include on the one hand the spaces of lattices and on the other Teichmüller and moduli spaces.

The classical kissing number problem is to bound the number of disjoint open unit balls that can be tangent to a fixed unit ball; the lattice kissing number is the same problem but where one asks that the centers of the spheres lie on some lattice. This is in fact an equivalent problem to counting the number of systoles (up to isotopy) of the underlying lattice. Another classical topic for flat tori is the study of Hermite constants. This involves finding sharp upper bounds on the length of shortest non-trivial lattice vectors or, in other words, bounds on the systole length of the quotient tori. Both of these problems make perfect sense for finite-area hyperbolic surfaces and have been studied by a variety of authors including Bavard [3] and Schmutz Schaller [17; 19].

Schmutz Schaller provided a variety of results on the length and the number of systoles for complete hyperbolic surfaces in both the closed and finite area cases. Lower bounds for either of these quantities can be found using arithmetic methods. Buser 
and Sarnak [8] were the first to show that there exist families $S_{k}$ of closed surfaces of genus $g_{k}$ with $g_{k} \rightarrow \infty$ as $k \rightarrow \infty$ whose systole length grows like

$$
\operatorname{sys}\left(S_{k}\right) \geq \frac{4}{3} \log g_{k} .
$$

Katz, Schaps and Vishne [11] generalized this construction to principal congruence subgroups of arbitrary arithmetic surfaces. Makisumi [12] showed that, in some sense, this is the best one can hope for via arithmetic constructions. Schmutz Schaller [19] found analogous results for kissing numbers: for any $\varepsilon>0$, there is a family of closed surfaces $T_{k}$ of genus $g_{k}$ with $g_{k} \rightarrow \infty$ as $k \rightarrow \infty$ whose number of systoles grows like

$$
\operatorname{Kiss}\left(T_{k}\right) \geq g_{k}^{\frac{4}{3}-\varepsilon}
$$

For surfaces with cusps, families reaching these lower bounds (for both quantities) are directly obtainable by considering principal congruence subgroups of $\mathrm{PSL}_{2}(\mathbb{Z})$ (see Schmutz Schaller [18], Brooks [5] and Balacheff, Makover and Parlier [2]). The number of cusps in these examples grows roughly like $g^{2 / 3}$.

Upper bounds for these quantities have also been studied, in particular for closed surfaces. Via an easy area argument, one can obtain an upper bound on the systole length of closed surfaces of genus $g$ that grows like $2 \log g$. This complements Buser and Sarnak's lower bound to show that the rough growth is logarithmic, but the discrepancy between the $\frac{4}{3}$ and the 2 remains mysterious. Schmutz Schaller, using a disk packing argument of Fejes Tóth, proved a very nice upper bound on systole length which is actually sharp for the congruence subgroups of $\mathrm{PSL}_{2}(\mathbb{Z})$ (see also Adams [1] and Bavard [4]). We use this result in an essential way and give the exact formulation in the sequel (Theorem 2.4).

For kissing numbers, the best known upper bounds are results of the second author [13]. In particular, there is a bound which depends only on the genus $g$ and which grows at most subquadratically in function of $g$. Again, there is a discrepancy between the $g^{4 / 3}$ lower bound and the $g^{2}$ upper bound (although the latter cannot be sharp). Upper bounds for kissing numbers of non-closed finite-area complete surfaces (ie surfaces with cusps) have yet to be approached. Filling this gap is the main goal of our article.

One of the main consequences of what we obtain is the following:

Theorem 4.11 There exists a universal constant $C>0$ such that, for any $S \in \mathcal{M}_{g, n}$, $g \geq 1$, its kissing number satisfies

$$
\operatorname{Kiss}(S) \leq C(g+n) \frac{g}{\log (g+1)}
$$


We obtain this result as a consequence of a number of results concerning the length and the topological configurations of systoles.

In particular, concerning the length of systoles, we show the following:

Theorem 2.3 There exists a universal constant $K<8$ such that every $S \in \mathcal{M}_{g, n}(g \neq 0)$ satisfies

$$
\operatorname{sys}(S) \leq 2 \log g+K \text {. }
$$

The result is not surprising in view of the results for closed surfaces and Schmutz Schaller's bound, but it is interesting to note that it is asymptotically a stronger bound when the growth of the number of cusps is bounded above by $g^{1 / 2}$.

Our results on topological configurations of systoles can be summarized as follows:

Propositions 3.2 and 3.3 and Lemma 3.5 If $\alpha$ and $\beta$ are systoles of a surface $S \in \mathcal{M}_{g, n}$, then

$$
i(\alpha, \beta) \leq 2
$$

and, if $i(\alpha, \beta)=2$, then either $\alpha$ or $\beta$ surrounds two cusps. Furthermore, for every genus $g \geq 0$, there exists $n(g) \in \mathbb{N}$ and a surface $S_{g}$ of genus $g$ with $n(g)$ cusps which has systoles that intersect twice.

The above result is in contrast with closed surfaces, where systoles can intersect at most once.

Finally, we obtain the following bound, which relates systolic lengths and kissing numbers.

Theorem 4.10 If $S \in \mathcal{M}_{g, n}$ has systole of length $\operatorname{sys}(S)=\ell$, then

$$
\operatorname{Kiss}(S) \leq 20 n \cosh \left(\frac{1}{4} \ell\right)+200 \frac{e^{\ell / 2}}{\ell}(2 g-2+n) .
$$

The article is organized as follows. In Section 2 we prove our upper bounds on systole length. Section 3 is dedicated to the study of the topological configurations of systoles. In Section 4 we prove Theorems 4.10 and 4.11.

Acknowledgements The authors would like to thank Bram Petri for useful discussions and the reviewer for her or his pertinent remarks and suggestions. Both authors were fully supported by Swiss National Science Foundation grant number PP00P2_128557. In addition, the authors acknowledge support from US National Science Foundation grants DMS 1107452, 1107263, 1107367 "RNMS: GEometric structures And Representation varieties" (the GEAR Network). The first author was also supported by Swiss National Science Foundation grant number P2FRP2_161723. 


\section{Bounds on lengths of systoles}

We denote by $\mathcal{M}_{g, n}$ the moduli space of surfaces of signature $(g, n)$, by which we mean the space of all complete finite area hyperbolic surfaces of genus $g$ with $n$ cusps up to isometry. We shall always assume that $g$ and $n$ satisfy $3 g-3+n>0$. A systole of a surface $S \in \mathcal{M}_{g, n}$ is a shortest closed geodesic. We think of systoles - and closed geodesics in general - as being non-oriented. Given a surface $S$, we denote its systole length (the length of one of its systoles) by $\operatorname{sys}(S)$. The main objective of this section is to show that every surface of genus $g \geq 1$, with or without cusps, has systole length bounded above by a function which only depends on the genus.

For any cusp $c$, let $H_{c}$ be the associated open horoball region of area 2. By the collar lemma (see for instance Chapter 4 of Buser [7]), two such regions are disjoint.

For any cusp $c$ and any non-negative $r$, define the set $D_{r}(c)$ to be

$$
D_{r}(c):=\left\{p \in S \mid d\left(p, H_{c}\right)<r\right\} \cup H_{c} .
$$

If $D_{r}(c)$ is homeomorphic to a once-punctured disk, we can compute its area, which is

$$
\operatorname{area}\left(D_{r}(c)\right)=2 e^{r} .
$$

Lemma 2.1 (a) If there are two cusps $c$ and $c^{\prime}$ such that $D_{r}(c)$ and $D_{r}\left(c^{\prime}\right)$ are tangent, then the simple closed geodesic forming a pair of pants with them has length $4 \operatorname{arccosh} e^{r}$, so

$$
\operatorname{sys}(S) \leq 4 \operatorname{arccosh}\left(e^{r}\right) .
$$

(b) If $D_{r}(c)$ is tangent to itself for some $r \geq \log 2$, then

$$
\operatorname{sys}(S) \leq 2 \operatorname{arccosh}\left(e^{r}-1\right) \text {. }
$$

Proof (a) Consider the pair of pants determined by the two cusps and the simple closed geodesic $\gamma$ surrounding them. Cut it along the orthogonal from $\gamma$ to itself, the shortest geodesic between the cusps and the perpendiculars from the cusps to $\gamma$. Consider one of the four obtained quadrilaterals; we denote its vertices by $q, s, t$ and $c$ and the intersection point of $\partial H_{c}$ with a side by $p$, as in Figure 1.

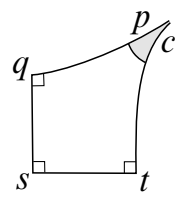

Figure 1: One of the quadrilaterals 


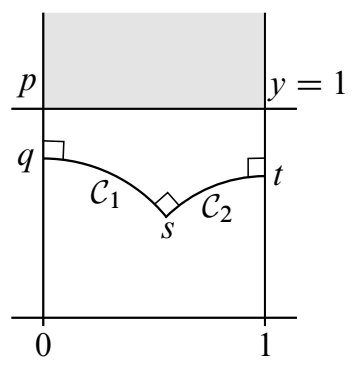

Figure 2: In the upper half-plane

Draw the quadrilateral in the upper half-plane, choosing infinity as the ideal point; see Figure 2. We fix the two geodesics containing $q c$ and $t c$ to be $x=0$ and $x=1$. The area of $H_{c}$ intersected with the quadrilateral is 1 , so $\partial H_{c}$ is given by $y=1$ and $p=i$. Moreover, $d(p, q)=\frac{1}{2} d\left(H_{c}, H_{c^{\prime}}\right)=r$, so $q=i e^{-r}$. Consider $\mathcal{C}_{1}$ and $\mathcal{C}_{2}$, the Euclidean circles representing the geodesics through $q$ and $s$ and through $s$ and $t$. Since $\mathcal{C}_{1} \perp\{x=0\}, \mathcal{C}_{2} \perp\{x=1\}$ and $\mathcal{C}_{1} \perp \mathcal{C}_{2}$, they have equations

$$
\begin{array}{rlrl}
\mathcal{C}_{1}: & x^{2}+y^{2} & =R^{2}, \\
\mathcal{C}_{2}: & (x-1)^{2}+y^{2}=1-R^{2}
\end{array}
$$

for some $R$. As $q \in \mathcal{C}_{1}$, we have $R=e^{r}$. By imposing $d(t, s)=\frac{1}{4} \ell$, we obtain $\ell=4 \operatorname{arccosh}\left(e^{r}\right)$.

(b) The cusp $c$ with the curve of length $2 r$ from $H_{c}$ and back determines a pair of pants with at least one simple closed geodesic as boundary.

If the pair of pants has two cusps and a boundary curve $\alpha$, we can cut it along the geodesic between the two cusps, the shortest geodesics between the cusps and $\alpha$ and the geodesic containing curve of length $2 r$. We get two right-angled triangles with two ideal vertices and $\frac{1}{2} \pi$ and two quadrilaterals with three right angles and an ideal vertex, as in Figure 3.

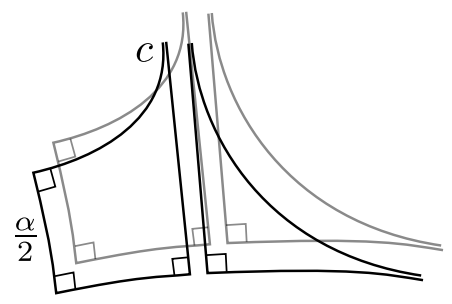

Figure 3: The cut pair of pants with two cusps 


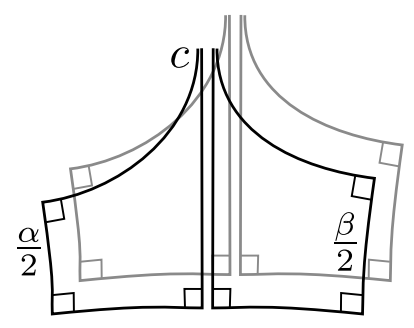

Figure 4: The cut pair of pants with one cusp

By direct computation similar to before, we obtain

$$
\ell(\alpha)=2 \operatorname{arccosh}\left(e^{r}-1\right) .
$$

If the pair of pants has two boundary curves, we denote them by $\alpha$ and $\beta$ and we suppose that $\ell(\alpha) \leq \ell(\beta)$. We cut along the orthogonal from $\alpha$ to $\beta$, the shortest geodesics from $\alpha$ and $\beta$ to the horoball and the geodesic containing the curve of length $2 r$. We obtain four quadrilaterals, with three right angles and an ideal vertex, pairwise isometric; see Figure 4.

Again by direct computation we have

$$
\begin{aligned}
& \ell(\alpha)=2 \operatorname{arccosh}\left(a e^{r}\right), \\
& \ell(\beta)=2 \operatorname{arccosh}\left((1-a) e^{r}\right),
\end{aligned}
$$

where $a$ is the area of $H_{c}$ intersected with one of the two quadrilaterals containing a part of $\alpha$. Since $\ell(\alpha) \leq \ell(\beta)$, we have $a \leq \frac{1}{2}$. Moreover, $\alpha$ is longest when $a$ is maximum, that is, when $a=\frac{1}{2}$. In this case

$$
\ell(\alpha)=\ell(\beta)=2 \operatorname{arccosh}\left(\frac{1}{2} e^{r}\right) .
$$

Since by assumption $r \geq \log 2$, we get that in both cases the curve $\alpha$ satisfies

$$
\ell(\alpha) \leq 2 \operatorname{arccosh}\left(e^{r}-1\right) .
$$

Remark 2.2 From the proof of the lemma we also have that, if $D_{r}(c)$ is tangent to itself for some $r \leq \log 2$, then $\operatorname{sys}(S) \leq 2 \operatorname{arcsinh} 1$.

We can now prove our bound on systole length for surfaces of genus $g \geq 1$.

Theorem 2.3 There exists a universal constant $K<8$ such that every $S \in \mathcal{M}_{g, n}$ satisfies

$$
\operatorname{sys}(S) \leq 2 \log g+K \text {. }
$$


Proof Set $\ell=\operatorname{sys}(S)$. We begin by recalling the well-known situation where $n=0$ (and thus $g \geq 2$ ). As the surface is closed, any open disk $D_{\ell / 2}(p)$ of radius $\frac{1}{2} \ell$ is embedded in the surface and thus

$$
\operatorname{area}\left(D_{\ell / 2}(p)\right)=2 \pi\left(\cosh \left(\frac{1}{2} \ell\right)-1\right) \leq \operatorname{area}(S)=2 \pi(2 g-2),
$$

which in turn implies

$$
\ell \leq 2 \log g+2 \log 4 .
$$

Suppose now that $n \geq 1$. We split the proof into three non-mutually exclusive cases. The first situation we consider is when there are "many" cusps (how many will be made explicit); in this case, two of the $D_{c}(r)$ have to meet for a "small" $r$ and will determine a short curve. In the second case, we assume that there are two cusps which are close to each other and the systole length will be bounded by the length of the curve surrounding them. In the final situation, there are "few" cusps and we further assume any two are far away; in this case, we show that there is a cusp with a short loop from its horoball to itself, which in turn determines a short curve.

\section{Case 1}

$$
n \geq \sqrt{2 \pi g} .
$$

If the sets $D_{r}(c)$ are pairwise disjoint for different cusps $c$ and each homeomorphic to a once-punctured disk, then

$$
\operatorname{area}\left(\bigcup_{c \text { cusp }} D_{r}(c)\right)=2 n e^{r} \leq \operatorname{area}(S)=2 \pi(2 g+n-2) ;
$$

thus,

$$
e^{r} \leq \frac{\pi(2 g-2+n)}{n} .
$$

Since $n \geq \sqrt{2 \pi g}$, this implies

$$
e^{r} \leq \frac{\sqrt{2 \pi}(g-1)}{\sqrt{g}}+\pi
$$

So, for some $r \leq \log (\sqrt{2 \pi}(g-1) / \sqrt{g}+\pi)$, either two $D_{r}(c)$ are tangent to each other or one is tangent to itself. Lemma 2.1 now implies

$$
\ell \leq 4 \operatorname{arccosh}\left(\frac{\sqrt{2 \pi}(g-1)}{\sqrt{g}}+\pi\right) .
$$

Case 2 There are distinct cusps $c_{1}$ and $c_{2}$ with $d\left(H_{c_{1}}, H_{c_{2}}\right) \leq \log (2 \pi(g-1+\sqrt{2 \pi g}))$. By Lemma 2.1,

$$
\ell \leq 4 \operatorname{arccosh}(\sqrt{2 \pi(g-1+\sqrt{2 \pi g})})
$$

and we are done. 
Case $30<n<\sqrt{2 \pi g}$ and any two cusps $c_{1}$ and $c_{2}$ satisfy

$$
d\left(H_{c_{1}}, H_{c_{2}}\right)>\log (2 \pi(g-1+\sqrt{2 \pi g})) .
$$

We fix a cusp $c$. Since any two cusps are far away, for $r \leq \log (2 \pi(g-1+\sqrt{2 \pi g}))$ the set $D_{r}(c)$ is disjoint from any other $H_{c^{\prime}}$. If it is also an embedded, once-punctured disk, then

$$
\operatorname{area}\left(D_{r}(c)\right)=2 e^{r} \leq \operatorname{area}(S)<4 \pi(g-1+\sqrt{2 \pi g})
$$

so

$$
r \leq \log (2 \pi(g-1+\sqrt{2 \pi g})) .
$$

We deduce that, for some $r \leq \log (2 \pi(g-1+\sqrt{2 \pi g})), D_{r}(c)$ is tangent to itself. By Remark 2.2, if $r \leq \log 2$ then $\ell \leq 2 \operatorname{arcsinh} 1$. Otherwise, by Lemma 2.1, we obtain

$$
\ell \leq 2 \operatorname{arccosh}(2 \pi(g-1+\sqrt{2 \pi g})-1) .
$$

Now any surface with $n>0$ will be in one of the three cases detailed above and, as such, we can deduce

$$
\begin{aligned}
\ell & \leq \max \{4 \operatorname{arccosh}(\sqrt{2 \pi}(g-1) / \sqrt{g}+\pi), 4 \operatorname{arccosh}(\sqrt{2 \pi(g-1+\sqrt{2 \pi g})}), \\
& 2 \operatorname{arccosh}(2 \pi(g-1+\sqrt{2 \pi g})-1)\} \\
& <2 \log g+8 .
\end{aligned}
$$

Applying the techniques of the above theorem to punctured spheres, one can show that the systole length of a punctured sphere is bounded by a uniform constant (which doesn't depend on the number of cusps). This is also a consequence of a theorem of Schmutz Schaller, who provided a different bound for the systole length of punctured surfaces.

Theorem 2.4 [17] For $S \in \mathcal{M}_{g, n}$ with $n \geq 2$ we have

$$
\operatorname{sys}(S) \leq 4 \operatorname{arccosh} \frac{6 g-6+3 n}{n} .
$$

For $n \sim g^{\alpha}$, Schmutz Schaller's bound grows roughly like $4(1-\alpha) \log g$. So our bound is stronger for $\alpha<\frac{1}{2}$, while Schmutz Schaller's is better for $\alpha \geq \frac{1}{2}$. 


\section{Intersection properties of systoles}

It is well known, via a simple cutting and pasting argument, that systoles on closed surfaces pairwise intersect at most once. On surfaces with cusps, this is not necessarily the case. For instance, on punctured spheres it is not difficult to see that systoles can intersect twice (the simplest case is a four-times punctured sphere with at least two systoles - they necessarily intersect and the minimal intersection number between two distinct curves is 2). This phenomenon also occurs for surfaces with positive genus. An example of this can be derived from Buser's hairy torus (see [7, Chapter 5]) with cusps instead of boundary curves and explicit examples in all genera are given in the sequel. On the other hand, since systole length is bounded within each moduli space, it follows from the collar lemma that the intersection number between any two systoles is also bounded. This can be considerably sharpened: the first main result of this section will be that two systoles on punctured surfaces can intersect at most twice.

We begin with some notation and well-known preliminary results. A curve is nontrivial if it represents a non-trivial element of the fundamental group. A non-trivial curve is essential if it does not bound a cusp. In particular, systoles are the shortest essential curves of a surface. Given two closed curves $\alpha$ and $\beta$, we denote by $i(\alpha, \beta)$ their geometric intersection number (the minimum number of transversal intersection points among representatives in the isotopy classes $[\alpha]$ and $[\beta]$ ). Two curves are said to intersect minimally if they intersect minimally among all representatives of their respective isotopy classes. The unique geodesics in the isotopy classes of simple closed curves are also simple and intersect minimally.

Let $\alpha$ and $\beta$ be simple closed geodesics on a surface $S$ with $i(\alpha, \beta) \geq 2$ and fix orientations on them. The curve $\alpha$ divides $\beta$ into $\operatorname{arcs}$ between consecutive intersection points. We say such an arc is of type I if the orientations at the two intersection points are different and of type II if the orientations are the same; see Figure 5.

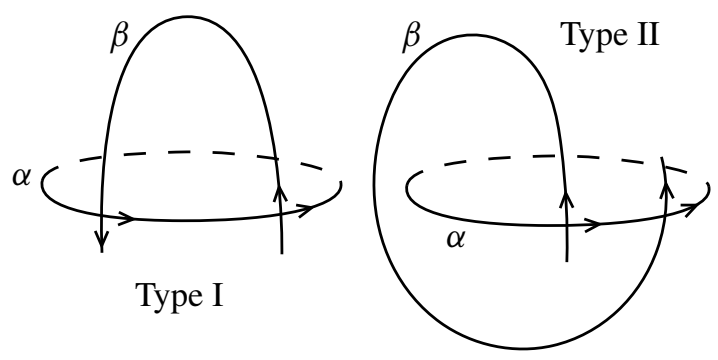

Figure 5: The two kinds of arcs 
Note that the orientation at each intersection point depends on the choice of orientations of $\alpha$ and $\beta$, but being of type I or II is independent of the choice of orientations.

Lemma 3.1 If $\alpha$ and $\beta$ are systoles of a surface $S \in \mathcal{M}_{g, n}$ with $i(\alpha, \beta) \geq 2$, all arcs between consecutive intersection points are of type $I$.

Proof By contradiction, suppose that $\beta$ contains arcs of type II. If there are at least two of them, there exists one, say $\beta_{1}$, of length at most $\frac{1}{2} \operatorname{sys}(S)$. Since $\beta_{1}$ divides $\alpha$ into two arcs, one of the two is of length at most $\frac{1}{2} \operatorname{sys}(S)$. Call this arc $\alpha_{1}$ and consider the curve $\alpha_{1} \cup \beta_{1}$.

If $\alpha_{1} \cup \beta_{1}$ were essential, its geodesic representative would be shorter than sys $(S)$, which is impossible. Thus $\alpha_{1} \cup \beta_{1}$ must be non-essential. However, one can construct a curve $\gamma$ homotopic to $\alpha_{1} \cup \beta_{1}$ such that $|\gamma \cap \alpha|=1$, so via the bigon criterion (see for instance Farb and Margalit [9]) $\gamma$ and $\alpha$ intersect minimally. Thus

$$
i(\gamma, \alpha)=1
$$

and as such $\gamma$ is non-trivial in homology and is therefore essential, a contradiction.

If there is exactly one arc $\beta_{1}$ of type II, there should be at least two (consecutive) arcs $\beta_{2}$ and $\beta_{3}$ of type I. Then, if $\ell\left(\beta_{1}\right) \leq \frac{1}{2} \operatorname{sys}(S)$, we can argue as before to obtain a contradiction. If not, then $\ell\left(\beta_{2} \cup \beta_{3}\right) \leq \frac{1}{2} \operatorname{sys}(S)$. The arcs $\beta_{2}, \beta_{3}$ and $\alpha$ determine an embedded four-holed sphere with a non-trivial curve of length at most $\operatorname{sys}(S)$. By construction, the geodesic in the isotopy class of this curve is strictly shorter than the systole, a contradiction.

Proposition 3.2 If $\alpha$ and $\beta$ are systoles of $S \in \mathcal{M}_{g, n}$, then $i(\alpha, \beta) \leq 2$.

Proof Suppose by contradiction that $i(\alpha, \beta)>2$. By Lemma 3.1, all arcs between consecutive intersection points are of type $\mathrm{I}$, so $i(\alpha, \beta)$ is even. Thus there are at least four intersection points and at least four arcs of $\beta$ between consecutive intersection points. This implies that there is an intersection point and two arcs $\beta_{1}$ and $\beta_{2}$ departing from it with $\ell\left(\beta_{1} \cup \beta_{2}\right) \leq \frac{1}{2} \operatorname{sys}(S)$. We argue as in the proof of Lemma 3.1: $\beta_{1}, \beta_{2}$ and $\alpha$ determine an embedded four-holed sphere with a non-trivial curve of length at most $\frac{1}{2} \operatorname{sys}(S)$. By construction, the geodesic in the isotopy class of this curve is strictly shorter than the systole, a contradiction.

The next proposition shows that if two systoles intersect twice, there is a constraint on the topological configuration of the two curves. 
Proposition 3.3 If two systoles $\alpha$ and $\beta$ intersect twice, one of them bounds two cusps.

Proof The two curves cut each other into arcs $\alpha_{1}, \alpha_{2}$ and $\beta_{1}, \beta_{2}$. Without loss of generality, we can assume $\ell\left(\alpha_{1}\right) \leq \ell\left(\beta_{1}\right) \leq \frac{1}{2} \operatorname{sys}(S)$. Consider $\gamma_{1}=\alpha_{1} \cup \beta_{1}$ and $\gamma_{2}=\alpha_{1} \cup \beta_{2}$. As $\gamma_{1}$ and $\gamma_{2}$ do not surround bigons, they cannot be trivial and, as they can be represented by curves of length strictly less than $\operatorname{sys}(S)$, they must both bound a cusp. Hence $\beta$ bounds two cusps.

An obvious consequence of Proposition 3.3 is that systoles on surfaces with at most one cusp intersect at most once. In the case of tori this can be improved to show that a surface with twice-intersecting systoles has at least three cusps.

Lemma 3.4 If $S \in \mathcal{M}_{1,2}$, and $\alpha$ and $\beta$ are systoles of $S$, then $i(\alpha, \beta) \leq 1$.

Proof Suppose two systoles $\alpha$ and $\beta$ intersect twice. Then $\operatorname{sys}(S) \geq 4 \operatorname{arcsinh} 1$ (see Gauglhofer and Semmler [10]) and, by Proposition 3.3, one of the two curves bounds two cusps. Cut the surface along $\alpha$ and consider the one-holed torus component. The length of the shortest closed geodesic $\gamma$ in the one-holed torus which doesn't intersect $\alpha$ satisfies (see Parlier [14])

$$
\cosh \left(\frac{1}{2} \ell(\gamma)\right) \leq \cosh \left(\frac{1}{6} \ell(\alpha)\right)+\frac{1}{2}
$$

and $\ell(\gamma) \geq \operatorname{sys}(S)=\ell(\alpha)$, so

$$
\cosh \left(\frac{1}{2} \ell(\alpha)\right) \leq \cosh \left(\frac{1}{6} \ell(\alpha)\right)+\frac{1}{2}
$$

which contradicts $\ell(\alpha) \geq 4 \operatorname{arcsinh} 1$.

On the other hand, we can prove that for every genus there is a punctured surface with systoles intersecting twice. The constructions will involve gluing ideal hyperbolic triangles. Any such triangle has a unique maximal embedded disk tangent to all three sides. We say that two such triangles are glued without shear if their embedded disks are tangent.

Lemma 3.5 For every $g \geq 0$, there exists $n(g) \in \mathbb{N}$ and a surface $S \in \mathcal{M}_{g, n(g)}$ with two systoles intersecting twice.

Proof For $g=0$, we can set $n(0)=4$, as mentioned at the beginning of Section 3: any four-times punctured sphere with at least two systoles will satisfy the requirement. To show the existence of such a surface, pick any $S \in \mathcal{M}_{0,4}$. If it has only one systole $\gamma$, 


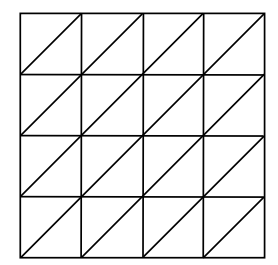

Figure 6: The triangulation of the square

increase the length of $\gamma$ so that it is still a systole and there is another simple closed geodesic on the surface of the same length.

For $g \geq 1$, we use a building block constructed as follows. Consider a square and a triangulation of it with 32 triangles, given by first subdividing the square into a grid of 16 squares and then adding one diagonal for all squares, as in Figure 6.

Each of the triangles in the square will be replaced by an ideal hyperbolic triangle and all gluings will be without shear.

For $g=1$, glue opposite sides of the square (again triangles are glued without shear) to obtain a torus with $n(1)=16$ cusps.

For $g \geq 2$, consider a polygon obtained by gluing a $1 \times(g-1)$ rectangle and a $1 \times 2(g-1)$ rectangle along the long sides, as in Figure 7 .

Think of this polygon as a $4 g$-gon (with sides corresponding to sides of the squares). Fix an orientation and choose a starting side, to identify the $4 g$ sides following the standard pattern $a_{1} b_{1} a_{1}^{-1} b_{1}^{-1} \cdots a_{g} b_{g} a_{g}^{-1} b_{g}^{-1}$ to obtain a genus- $g$ surface. If we now replace each $1 \times 1$ square by the building block (always gluing adjacent triangles without shear), we get a surface of genus $g$ with a decomposition into $32 \cdot 3(g-1)$ ideal triangles. Since it is a triangulation, the number of edges is $\frac{3}{2} \cdot 32 \cdot 3(g-1)$. By an Euler characteristic argument, this implies that the surface has $n(g)=46 g-46$ cusps.

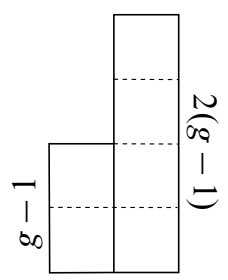

Figure 7: The polygon for $g=3$ 
For any $g \geq 1$, consider the set $\mathcal{C}_{g}$ of curves surrounding pairs of cusps which are connected by an edge between vertices of degree 6 in the triangulation of the surface. By construction, each of these intersects another such curve twice and we defer the proof that these curves are systoles to Lemma 3.6.

We now prove our claim that the curves in $\mathcal{C}_{g}$ are indeed systoles.

Lemma 3.6 For all $g \geq 1$, the curves in $\mathcal{C}_{g}$ are systoles.

Proof Consider the triangulation of the surface. For $g=1$, all vertices are of degree 6 . When $g \geq 2$, the pasting scheme associates all exterior vertices of the $4 g$-gon and the point in the quotient has degree $12 g-6$ (to see this, simply apply the hand-shaking lemma to the graph given by the triangulation). The remaining vertices are all of degree 6 . We denote by $\Gamma$ the graph dual to the triangulation. From what we have just said, for $g=1$, cutting the surface along $\Gamma$ decomposes the surface into hexagons. When $g \geq 2$, cutting along $\Gamma$ decomposes the surface into hexagons and a single $(12 g-6)-$ gon.

Any simple closed oriented geodesic $\gamma$ on the surface can be homotoped to a curve on $\Gamma$. At every vertex crossed by the curve, the orientations on the surface and on the curve give us a notion of "going left" or "going right". We can associate to $\gamma$ a word $w$ in the matrices $L=\left(\begin{array}{ll}1 & 1 \\ 0 & 1\end{array}\right)$ and $R=\left(\begin{array}{ll}1 & 0 \\ 1 & 1\end{array}\right)$, where each $L$ corresponds to a left turn and each $R$ to a right turn. This way of understanding curves on "zero shear surfaces" is fully explained in [6]. In particular, Brooks and Makover show how to compute the length of these curves in terms of the associated word:

$$
\ell(\gamma)=2 \operatorname{arccosh}\left(\frac{1}{2} \operatorname{Tr}(w)\right) .
$$

Each curve in $\mathcal{C}_{g}$ corresponds to the word $w_{0}=R L^{4} R L^{4}$ (or $L R^{4} L R^{4}$ or any cyclic permutation of these, depending on the choice of an orientation and a starting point on the curve), which, via a simple computation, has trace 34 . To show that the curves in $\mathcal{C}_{g}$ are systoles, it is enough to show that all other words corresponding to simple closed geodesics have trace at least 34 .

We use the following remark (see for instance [15]):

Remark 3.7 If a word can be written as $w=\cdots w_{1} \cdots w_{2} \cdots w_{k} \cdots$, then

$$
\operatorname{Tr}(w) \geq \operatorname{Tr}\left(w_{\sigma(1)} \cdots w_{\sigma(k)}\right)
$$

for any cyclic permutation $\sigma$ of $1, \ldots, k$. 
Let $\gamma$ be a simple closed geodesic which is not in $\mathcal{C}_{g}$. First we observe that we only need to consider curves represented by circuits in $\Gamma$. Indeed, if $\gamma$ corresponds to a closed path which contains an essential (ie not corresponding to a curve going around a cusp) circuit $\gamma^{\prime}$, a word representing $\gamma$ will contain a word representing $\gamma^{\prime}$. By Remark 3.7, $\gamma^{\prime}$ is at most as long as $\gamma$ and we can consider $\gamma^{\prime}$ instead. Otherwise, if $\gamma$ is formed from non-essential circuits, it should contain at least two of them. Note that, since non-essential circuits surround a cusp, they trace a hexagon or a $(12 g-6)$-gon. If both these circuits surround hexagons, we are in one of the following situations:

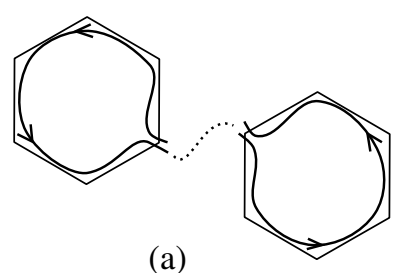

(a)

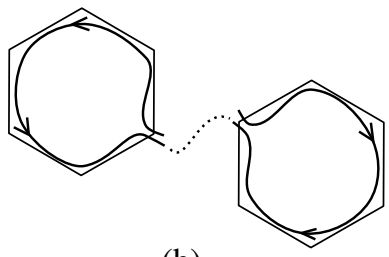

(b)

In case (a) a word associated to the curve contains $R L^{5} \cdots R L^{5}$ and in case (b) it contains $L R^{5} \cdots R L^{5}$. In both cases, by Remark 3.7 and a simple computation, their traces are bigger than 34 . Now, if one of the two circuits surrounds the vertex of the triangulation of degree $12 g-6$, the curve is even longer.

Suppose then that $\gamma$ is represented by an essential circuit. If it passes through five consecutive edges of a hexagon (said differently, a corresponding word contains $L^{4}$ ) and is not in $\mathcal{C}_{g}$, the following modification of the curve (see Figure 8) provides an essential circuit. A word of the curve on the left contains $L R^{4} L$, while the one of the curve on the right contains $R^{2}$, so the trace decreases (again by Remark 3.7) and we obtain a shorter curve.

We now assume a word $w$ representing $\gamma$ does not contain $L^{4}$ or $R^{4}$ and as such it is made of blocks of type $L^{i} R^{j}$ for $1 \leq i, j \leq 3$. If $w$ is made of four or more such blocks, then

$$
\operatorname{Tr} w \geq \operatorname{Tr}\left((L R)^{4}\right)>34 .
$$

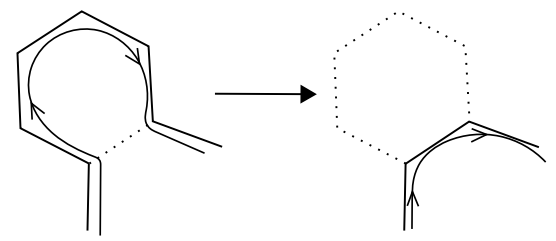

Figure 8: Shortening a curve 
Moreover, the length of $w$ is at least 7, as the shortest circuits in $\Gamma$ are of length 6 and correspond to curves surrounding cusps. With this in hand, one needs to check the finite set of words $w$ made of blocks as above, of length at least 7 , and of trace at most 33. To do this one can proceed as follows. The conditions on $w$ give two systems of equations for the exponents of $L$ and $R$ (a system for the words made of two blocks as above and one for words made of three blocks). These systems can be solved to get the set of words we are interested in. It is then straightforward to check that the curves corresponding to these words do not correspond to simple closed geodesics on the surface.

\section{Kissing number bound}

In this section we will prove an upper bound for the kissing number depending on the systole length. We then deduce a universal upper bound depending only on the signature of the surface. To do so, we separate the systoles into three sets and we give separate bounds for each of their cardinalities.

For a surface $S$, let $\mathfrak{S}(S)$ be the set of its systoles and $\operatorname{Kiss}(S):=|\mathfrak{S}(S)|$ be the kissing number of $S$. We say that $\alpha$ and $\beta$ bound a cusp if they form a pair of pants with a cusp. We define:

$$
\begin{aligned}
& A(S):=\{\alpha \in \mathfrak{S}(S) \mid \alpha \text { bounds two cusps }\} . \\
& B(S):=\{\alpha \in \mathfrak{S}(S) \backslash A(S) \mid \alpha \text { and } \beta \text { bound a cusp for some } \beta \in \mathfrak{S}(S) \backslash A(S)\} . \\
& C(S):=\mathfrak{S}(S) \backslash(A(S) \cup B(S)) .
\end{aligned}
$$

Note that by Proposition 3.3 two systoles in $\mathfrak{S}(S) \backslash A(S)$ intersect at most once.

\subsection{Bounds on $|A(S)|$}

As seen in Lemma 2.1, a curve of length $\ell$ bounds two cusps $c$ and $c^{\prime}$ if and only if the distance between $H_{c}$ and $H_{c^{\prime}}$ is

$$
d(\ell)=2 \log \cosh \left(\frac{1}{4} \ell\right),
$$

To bound $|A(S)|$ we will bound the number of pairs of cusps at distance $d(\operatorname{sys}(S))$.

Lemma 4.1 Let $S$ be a surface with $\operatorname{sys}(S)=\ell$ and $c$ a cusp of $S$. There are at most $\left\lfloor 2 \cosh \left(\frac{1}{4} \ell\right)\right\rfloor \operatorname{cusps} c^{\prime}$ which satisfy $d\left(H_{c}, H_{c^{\prime}}\right)=d(\ell)$. 
Proof Suppose $c_{1}$ and $c_{2}$ are two cusps such that

$$
d\left(H_{c}, H_{c_{1}}\right)=d\left(H_{c}, H_{c_{2}}\right)=d(\ell) .
$$

Since $\operatorname{sys}(S)=\ell$, the distance between $H_{c_{1}}$ and $H_{c_{2}}$ is at least $d(\ell)$. Consider

- the segment $\alpha$ realizing the distance between $H_{c}$ and $H_{c_{1}}$,

- the segment $\beta$ realizing the distance between $H_{c}$ and $H_{c_{2}}$,

- the shortest arc $\gamma$ of $\partial H_{c}$ bounded by the endpoints of $\alpha$ and $\beta$.

Let $\delta$ be the unique geodesic segment freely homotopic with endpoints on $\partial H_{c_{1}}$ and $\partial H_{c_{2}}$ to the curve $\alpha \cup \beta \cup \gamma$. Then its length is at least $d(\ell)$ :

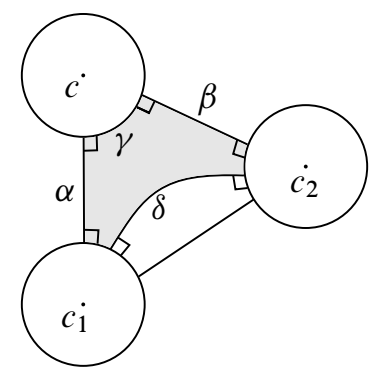

By a direct computation on the (non-geodesic) hexagon determined by $\alpha, \beta, \delta$ and the three horocycles, one can show that

$$
\ell(\gamma) \geq \frac{1}{\cosh \left(\frac{1}{4} \ell\right)} .
$$

Since $\partial H_{c}$ has length 2 , the number of cusps around $c$ at distance $d(\ell)$ is bounded above by

$$
\frac{2}{1 / \cosh \left(\frac{1}{4} \ell\right)},
$$

which proves the claim as we are bounding an integer.

As a consequence, we get the following:

Proposition 4.2 For $S \in \mathcal{M}_{g, n}$ with $\operatorname{sys}(S)=\ell$,

$$
|A(S)| \leq \frac{1}{2} n\left\lfloor 2 \cosh \left(\frac{1}{4} \ell\right)\right\rfloor .
$$

Proof There are $n$ cusps, each of which can be surrounded by at most $\left\lfloor 2 \cosh \left(\frac{1}{4} \ell\right)\right\rfloor$ cusps at distance $d(\ell)$. The result follows as each curve surrounds two cusps. 
Remark 4.3 We can get another upper bound for $A(S)$ using the Euler characteristic as follows.

Consider the set of punctures; if there is a systole bounding two of them, we join them with a simple geodesic lying in the pair of pants determined by the systole. We complete this set of geodesics into an ideal triangulation (decomposition into ideal triangles) of the surface. The number of vertices of the triangulation is the number of punctures $n$. If $e$ is the number of edges, the number of triangles is $\frac{2}{3} e$. The Euler characteristic of the compactified surface is $2 g-2$, so

$$
n-e+\frac{2}{3} e=2-2 g \text {. }
$$

From how we constructed the triangulation, it is clear that $|A(S)| \leq e$, so we get

$$
|A(S)| \leq 3(n+2 g-2) .
$$

Interestingly, this bound can also be seen as a corollary of the above proposition. If we use Schmutz Schaller's upper bound on systole length (Theorem 2.4) in Proposition 4.2 above, this is exactly the resulting bound.

For surfaces of genus at least one, we will use the bound from the remark above, but for punctured spheres we will use Proposition 4.2 directly.

\subsection{Bound on $|B(S)|$}

Consider a cusp $c$; we define two associated sets:

$$
\begin{aligned}
B(c) & :=\{\alpha \in B(S) \mid \alpha \text { and } \beta \text { bound } c \text { for some } \beta \in B(S)\}, \\
B(c)^{(2)} & :=\{(\alpha, \beta) \in B(S) \times B(S) \mid \alpha \text { and } \beta \text { bound } c\} .
\end{aligned}
$$

Suppose $(\alpha, \beta),(\gamma, \delta) \in B(c)^{(2)}$. Then $\gamma$ has to pass through the pair of pants given by $\alpha, \beta$ and $c$, so $\gamma$ must intersect $\alpha$ or $\beta$. Since curves in $\mathfrak{S}(S) \backslash A(S)$ pairwise intersect at most once, $i(\alpha, \gamma)=i(\beta, \gamma)=1$ (and the same for $\delta$ ).

Any curve $\alpha \in B(c)$ is at a fixed distance $D(\ell)$ from $H_{c}$. By a direct computation in the pair of pants bounded by $\alpha$ and $\beta$, one obtains

$$
D(\ell)=\log \left(2 \frac{\cosh \left(\frac{1}{2} \ell\right)}{\sinh \left(\frac{1}{2} \ell\right)}\right) .
$$

When curves in $B(S)$ intersect they do so exactly once and we can obtain a lower bound on their angle of intersection of curves in $B(S)$. (Note that the lemma holds for any pair of systoles that intersect once.) 


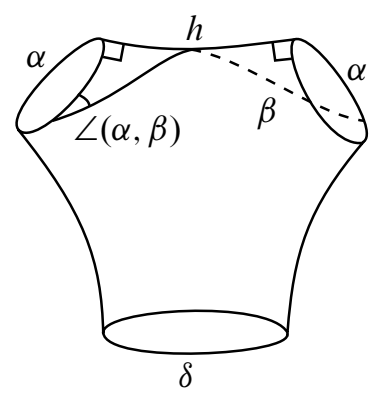

Figure 9: The result of cutting the torus $T$ along $\alpha$

Lemma 4.4 Let $S$ be a surface of signature $(g, n) \neq(1,1)$. If $\alpha$ and $\beta$ are systoles of length $\ell$ intersecting once, their angle of intersection satisfies

$$
\sin \angle(\alpha, \beta) \geq \sin \theta_{\ell}:= \begin{cases}2 / \sqrt{5}, & \ell<2 \operatorname{arccosh} \frac{3}{2}, \\ \sqrt{2 \cosh \left(\frac{1}{2} \ell\right)+1} /\left(\cosh \left(\frac{1}{2} \ell\right)+1\right), & \ell \geq 2 \operatorname{arccosh} \frac{3}{2}\end{cases}
$$

In particular, the angle of intersection is bounded below by a function $\theta_{\ell}$ that behaves like $e^{-\ell / 4}$ as $\ell$ goes to infinity.

Note that [13, Lemma 2.4] also gives a lower bound on the angle of intersection, with the same order of growth.

Proof Consider the two systoles and the one-holed torus $T$ they determine. Since $(g, n) \neq(1,1)$, the boundary component $\delta$ of $T$ is a simple closed geodesic.

As $\alpha$ and $\beta$ are systoles of $S$, they are also systoles of $T$. As such they satisfy the systole bound for $T$ that depends on the length of $\delta$, namely

$$
\cosh \left(\frac{1}{6} \ell(\delta)\right) \geq \cosh (\ell)-\frac{1}{2} \text {. }
$$

We first consider the case when $\ell \geq 2 \operatorname{arccosh} \frac{3}{2}$. We have $\cosh \left(\frac{1}{2} \ell\right)-\frac{1}{2} \geq 1$ and the condition stated above is non-empty. Cut $T$ along $\alpha$ (see Figure 9) and consider the shortest curve $h$ connecting the two copies of $\alpha$. By hyperbolic trigonometry, using $\cosh \left(\frac{1}{6} \ell(\delta)\right) \geq \cosh \left(\frac{1}{2} \ell\right)-\frac{1}{2}$, a direct computation provides

$$
\cosh h \geq \frac{4 \cosh \left(\frac{1}{2} \ell\right)^{2}-\cosh \left(\frac{1}{2} \ell\right)-1}{\cosh \left(\frac{1}{2} \ell\right)+1} .
$$

Now consider one of the two right-angled triangles determined by $\operatorname{arcs}$ of $\alpha, \beta$ and $h$. We have

$$
\frac{\sinh \left(\frac{1}{2} h\right)}{\sin \angle(\alpha, \beta)}=\sinh \left(\frac{1}{2} \ell\right)
$$




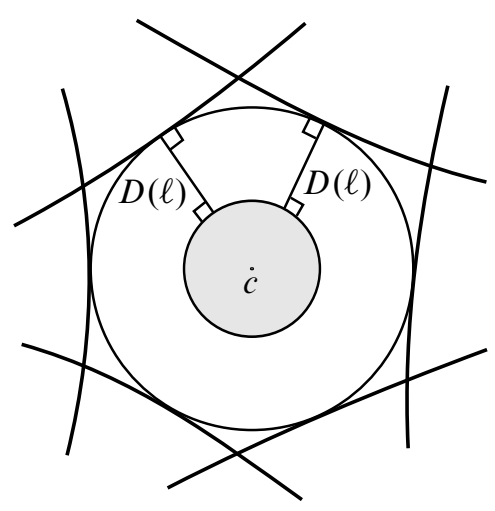

Figure 10: Geodesics around a horoball

which, together with the estimate on $h$, yields

$$
\sin \angle(\alpha, \beta) \geq \frac{\sqrt{2 \cosh \left(\frac{1}{2} \ell\right)+1}}{\cosh \left(\frac{1}{2} \ell\right)+1} .
$$

If $\ell<2 \operatorname{arccosh} \frac{3}{2}$, we deduce the inequality $\sin \angle(\alpha, \beta) \geq 2 / \sqrt{5}$ by arguing as above, but replacing the estimate $\cosh \left(\frac{1}{6} \ell(\delta)\right) \geq \cosh \left(\frac{1}{2} \ell\right)-\frac{1}{2}$ by $\ell(\delta) \geq \ell$.

Fix $(\alpha, \beta) \in B(c)^{(2)}$ and denote by $\mathcal{P}$ the pair of pants they determine with $c$. As they form a pair of pants with two boundary curves of the same length, there is an isometric involution $\varphi$ of $\mathcal{P}$ that sends $\alpha$ to $\beta$ (a rotation of angle $\pi$ around the cusp). Note that, for any $(\gamma, \delta) \in B(c)^{(2)}$, the involution sends $\gamma \cap \mathcal{P}$ to $\delta \cap \mathcal{P}$ because of the symmetry of the pair of pants determined by $\gamma, \delta$ and $p$. If we quotient $\mathcal{P}$ by $\varphi$ and we consider the image of $B(c)$, we get a set of geodesics at distance $D(\ell)$ from a horoball of area 1 , all pairwise intersecting with angle at least $\theta_{\ell}$. This observation is crucial to show the following result.

Lemma 4.5 If $(g, n) \neq(1,1)$, the number of elements in $B(c)$ is bounded above by

$$
m(\ell):=\frac{\cosh \left(\frac{1}{2} \ell\right)}{\sinh \left(\frac{1}{2} \ell\right)} \frac{2}{\sin \left(\frac{1}{2} \theta_{\ell}\right)} .
$$

Proof The situation is as in Figure 10, which locally represents the elements of $B(c)$ under the quotient by $\varphi$. Note that every element in the quotient by $\varphi$ represents two elements from $B(c)$.

The inner circle (which we'll refer to as the inner horocycle) represents the quotient horoball of area 1 and the external one is the horocycle at distance $D(\ell)$ from the 
horoball of area 1. By looking at the unique orthogonal geodesics between elements of $B(c) / \varphi$ and the inner horocycle, we can determine a cyclic ordering on the elements of $B(c) / \varphi$. Two neighboring geodesics with respect to this ordering determine a subarc on the inner horocycle as follows. We consider the orthogonal geodesic between them and the inner horocycle and take the subarc of the horocycle which forms a pentagon with the two geodesics and the orthogonal (see Figure 10). By a direct computation, using the lower bound on the angle of intersection, this subarc on the inner horocycle is of length at least

$$
\frac{\sinh \left(\frac{1}{2} \ell\right)}{\cosh \left(\frac{1}{2} \ell\right)} \sin \left(\frac{1}{2} \theta_{\ell}\right) \text {. }
$$

These subarcs are all disjoint and are of the same number as the elements of $B(c) / \varphi$ (keep in mind that any two elements of $B(c) / \varphi$ intersect).

From this we deduce an upper bound on $|B(c) / \varphi|$ :

$$
\frac{1}{\left(\sinh \left(\frac{1}{2} \ell\right) / \cosh \left(\frac{1}{2} \ell\right)\right) \sin \left(\frac{1}{2} \theta_{\ell}\right)} \text {. }
$$

Now $2|B(c) / \varphi|=|B(c)|$, which completes the proof.

As a consequence, we obtain an upper bound on $|B(S)|$.

Proposition 4.6 If $S \in \mathcal{M}_{g, n},(g, n) \neq(1,1)$, has systole of length $\operatorname{sys}(S)=\ell$, then

$$
|B(S)| \leq n m(\ell) .
$$

Proof We have

$$
B(S)=\bigcup_{c \text { cusp }} B(c)
$$

and, for every cusp $c$,

$$
|B(c)| \leq m(\ell) .
$$

\subsection{Bound on $|C(S)|$}

By definition, elements of $C(S)$ are systoles that satisfy

- two curves in $C(S)$ intersect at most once, and

- two disjoint curves in $C(S)$ do not bound a cusp.

We follow a similar argument to one found in [13] to obtain an upper bound on $|C(S)|$. In particular, we will need a collar lemma for systoles. 
Lemma 4.7 Let $\operatorname{sys}(S)=\ell$ and consider $\alpha, \beta \in C(S)$. If $\alpha$ and $\beta$ do not intersect, then they are at distance at least $2 r(\ell)$, where

$$
r(\ell)=\operatorname{arcsinh} \frac{1}{2 \sinh \left(\frac{1}{4} \ell\right)} .
$$

Proof Fix a pair of pants with $\alpha$ and $\beta$ as boundary and consider the third boundary component $\gamma$. Since $\alpha$ and $\beta$ are in $C(S)$, they do not bound a cusp, so $\gamma$ is a simple closed geodesic of length at least $\ell$. The result follows by a standard trigonometric computation.

As a consequence, if $\alpha$ and $\beta$ in $C(S)$ pass through the same disk of radius $r(\ell)$ then they intersect.

Moreover, we have seen in Lemma 4.4 that there is a lower bound on the angle of intersection of systoles intersecting once. With this in hand we prove the following:

Lemma 4.8 If $(g, n) \neq(1,1)$, $\operatorname{sys}(S)=\ell$, and $\alpha$ and $\beta$ in $C(S)$ pass through a disk of center $p$ and radius $r(\ell)$, then the distance between $p$ and the point $q$ of intersection between $\alpha$ and $\beta$ satisfies

$$
d(p, q) \leq R(\ell)
$$

where

$$
\sinh (R(\ell))= \begin{cases}5 /\left(8 \sinh \left(\frac{1}{4} \ell\right)\right), & \ell<2 \operatorname{arccosh} \frac{3}{2}, \\ \left(\cosh \left(\frac{1}{2} \ell\right)+1\right) /\left(2 \sinh \left(\frac{1}{4} \ell\right) \sqrt{\left.2 \cosh \left(\frac{1}{2} \ell\right)+1\right)},\right. & \ell \geq 2 \operatorname{arccosh} \frac{3}{2} .\end{cases}
$$

Note that $R(\ell)$ is bounded for $\ell \geq 2 \operatorname{arcsinh} 1$.

Proof The proof is analogous to the proof of [13, Lemma 2.6]. Fix $p_{\alpha} \in \alpha$ and $p_{\beta} \in \beta$ lying in $D_{r(\ell)}(p)$. We have two triangles of vertices $p, p_{\alpha}, q$ and $p, p_{\beta}, q$, and the sum of the two angles $\theta_{\alpha}$ and $\theta_{\beta}$ at $q$ is the angle of intersection $\angle(\alpha, \beta)$. Suppose $\theta_{\alpha} \geq \frac{1}{2} \angle(\alpha, \beta)$ and consider the angle $\eta$ of the triangle $p, p_{\alpha}, q$ at $p_{\alpha}$; see Figure 11 . Then

$$
\frac{\sin (\eta)}{\sinh (d(p, q))}=\frac{\sin \left(\theta_{\alpha}\right)}{\sinh \left(d\left(p, p_{\alpha}\right)\right)} \text {. }
$$

Using $\theta_{\alpha} \geq \frac{1}{2} \angle(\alpha, \beta), d\left(p, p_{\alpha}\right)<r(\ell)$ and Lemma 4.4, we obtain the result.

We are now in a position to obtain a bound on $|C(S)|$.

Proposition 4.9 If $S \in \mathcal{M}_{g, n}, g \neq 0$ and $(g, n) \neq(1,1)$, has systole of length $\operatorname{sys}(S)=\ell$, then

$$
|C(S)| \leq 200 \frac{e^{\ell / 2}}{\ell}(2 g-2+n) .
$$




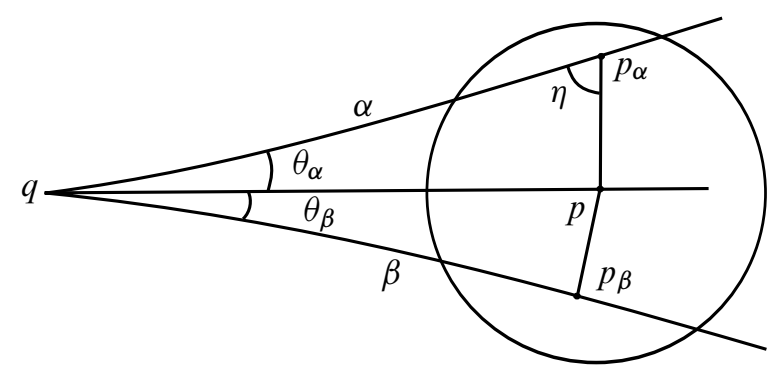

Figure 11: $\alpha$ and $\beta$ passing though a disk of radius $r(\ell)$

Proof If $\ell \leq 2 \operatorname{arcsinh} 1$, then all systoles are pairwise disjoint, so

$$
|C(S)| \leq \operatorname{Kiss}(S) \leq 3 g-3+n .
$$

We now suppose that $\ell>2 \operatorname{arcsinh} 1$. Consider $\tilde{S}=S \backslash \bigcup_{c \text { cusp }} D_{w(\ell)}(c)$, where

$$
w(\ell)=\operatorname{arcsinh} \frac{1}{\sinh \left(\frac{1}{2} \ell\right)}
$$

is the width of a collar around a systole. By the collar lemma, each curve of $C(S)$ is contained in $\tilde{S}$. We cover $\tilde{S}$ with disks of radius $r(\ell)$. Then the cardinality of $C(S)$ is bounded above by

$$
\frac{F(S) G(S)}{H(S)}
$$

where

$$
\begin{aligned}
& F(S)=\#\{\text { balls of radius } r(\ell) \text { needed to cover } \tilde{S}\}, \\
& G(S)=\#\{\text { curves in } C(S) \text { crossing a ball of radius } r(\ell)\}, \\
& H(S)=\#\{\text { number of balls of radius } r(\ell) \text { a curve in } C(S) \text { must cross }\} .
\end{aligned}
$$

To bound $|C(S)|$, we need to give upper bounds for $F(S)$ and $G(S)$ and a lower bound for $H(S)$.

\section{Upper bound for $\boldsymbol{F}(\boldsymbol{S})$ We have}

$F(S) \leq \max \#\left\{\right.$ embedded balls of radius $\frac{1}{2} r(\ell)$ which are pairwise disjoint $\}$

$$
\leq \frac{\operatorname{area}(\tilde{S})}{\operatorname{area}\left(\text { ball of radius } \frac{1}{2} r(\ell)\right)} \leq \frac{\operatorname{area}(S)}{2 \pi\left(\cosh \left(\frac{1}{2} r(\ell)\right)-1\right)} \leq 8(2 g-2+n) e^{\ell / 2} .
$$

Upper bound for $\boldsymbol{G}(\boldsymbol{S})$ We proceed as in the proof of [13, Theorem 2.9], by reasoning in the universal cover and estimating how many geodesics, pairwise intersecting at 
an angle of at least $\theta_{\ell}$, can intersect a disk of radius $r(\ell)$. We obtain

$$
G(S) \leq \frac{\pi}{2} \frac{\sinh (R(\ell)+\operatorname{arcsinh} 1)}{\operatorname{arcsinh} \sin \left(\theta_{\ell}\right)} \leq \frac{5 \pi}{2 \operatorname{arcsinh} \sin \left(\theta_{\ell}\right)} .
$$

Lower bound for $\boldsymbol{H}(\boldsymbol{S})$ To cover a curve of length $\ell$ with disks of radius $r(\ell)$ we need at least $\ell /(2 r(\ell))$. So

$$
H(S) \geq \frac{\ell}{2 \operatorname{arcsinh}\left(1 /\left(2 \sinh \left(\frac{1}{4} \ell\right)\right)\right)} \geq \ell \sinh \left(\frac{1}{4} \ell\right) .
$$

By putting the three bounds together and considering that $\sinh \left(\frac{1}{4} \ell\right) \operatorname{arcsinh} \sin \left(\theta_{\ell}\right)$ is bounded below by $\frac{1}{3}$ for $\ell>2 \operatorname{arcsinh} 1$ we obtain the claimed result.

\subsection{Proof of main results}

Using Propositions 4.2, 4.6 and 4.9, we get an upper bound for the kissing number of a surface in terms of its signature and its systole length.

Theorem 4.10 If $S \in \mathcal{M}_{g, n}$ with $g \geq 1,(g, n) \neq(1,1)$, has systole of length $\operatorname{sys}(S)=\ell$, then

$$
\operatorname{Kiss}(S) \leq 20 n \cosh \left(\frac{1}{4} \ell\right)+200 \frac{e^{\ell / 2}}{\ell}(2 g-2+n) .
$$

As a consequence, we can get a bound on the kissing number which is independent of the systole length.

Theorem 4.11 There exists a universal constant $C$ (which we can take to be $2 \times 10^{4}$ ) such that, for any $S \in \mathcal{M}_{g, n}, g \geq 1$, its kissing number satisfies

$$
\operatorname{Kiss}(S) \leq C(g+n) \frac{g}{\log (g+1)}
$$

Proof This follows from the bounds in Theorem 4.10 and bounds on systole lengths. Precisely, we insert the Schmutz Schaller bound (Theorem 2.4) into the term $\cosh \left(\frac{1}{4} \ell\right)$ and we use Theorem 2.3 for the $e^{\ell / 2} / \ell$ term. For $(g, n)=(1,1)$, we recall the wellknown fact that $\operatorname{Kiss}(S) \leq 3$ (there can be at most 3 distinct curves that pairwise intersect at most once on a one-holed torus).

Remark 4.12 Przytycki [16] obtained an upper bound for the number of simple closed curves pairwise intersecting at most once. Using this and our bound on $|A(S)|$ (Proposition 4.2), one can obtain an upper bound for the kissing number which is cubic in the Euler characteristic. Our upper bound, on the other hand, is subquadratic in $|\chi(S)|$, like the one for closed surfaces in [13]. 
The upper bound of Theorem 4.11 is linear in the number of cusps if we fix the genus. For punctured spheres we can obtain a more meaningful bound.

Theorem 4.13 For every $S \in \mathcal{M}_{0, n}$, the number of systoles satisfies

$$
\operatorname{Kiss}(S) \leq \frac{7}{2} n-5 \text {. }
$$

Proof By Proposition 4.2 and Schmutz Schaller's upper bound for the systole, we have

$$
|A(S)| \leq \frac{n}{2}\left\lfloor\frac{2(3 n-6)}{n}\right\rfloor=\frac{n}{2}\left\lfloor 6-\frac{12}{n}\right\rfloor \leq \frac{5}{2} n .
$$

Moreover, systoles are separating, so can only intersect an even number of times. This implies that systoles in $\mathfrak{S}(S) \backslash A(S)$ are pairwise disjoint and hence part of a pants decomposition. Note that any pants decomposition of a sphere contains at least two curves bounding two cusps; indeed, the dual graph to the pants decomposition is a tree, so it has at least two leaves, which correspond to curves bounding two cusps. This implies that

$|\mathfrak{S}(S) \backslash A(S)| \leq \#$ curves in a pants decomposition $-2=n-5$.

By using short pants decompositions where every curve is of equal length, it is easy to obtain a family of punctured spheres with a number of systoles that grows linearly in the number of cusps. Matching the $\frac{7}{2} n$ upper bound from this theorem seems much more challenging.

\section{References}

[1] C Adams, Maximal cusps, collars, and systoles in hyperbolic surfaces, Indiana Univ. Math. J. 47 (1998) 419-437 MR1647904

[2] F Balacheff, E Makover, H Parlier, Systole growth for finite area hyperbolic surfaces, Ann. Fac. Sci. Toulouse Math. 23 (2014) 175-180 MR3204736

[3] C Bavard, Systole et invariant d'Hermite, J. Reine Angew. Math. 482 (1997) 93-120 MR1427658

[4] C Bavard, Anneaux extrémaux dans les surfaces de Riemann, Manuscripta Math. 117 (2005) 265-271 MR2154250

[5] R Brooks, Platonic surfaces, Comment. Math. Helv. 74 (1999) 156-170 MR1677565

[6] R Brooks, E Makover, Random construction of Riemann surfaces, J. Differential Geom. 68 (2004) 121-157 MR2152911

[7] P Buser, Geometry and spectra of compact Riemann surfaces, Progress in Mathematics 106, Birkhäuser, Boston (1992) MR1183224 
[8] P Buser, P Sarnak, On the period matrix of a Riemann surface of large genus, Invent. Math. 117 (1994) 27-56 MR1269424

[9] B Farb, D Margalit, A primer on mapping class groups, Princeton Mathematical Series 49, Princeton Univ. Press (2012) MR2850125

[10] T Gauglhofer, K-D Semmler, Trace coordinates of Teichmüller space of Riemann surfaces of signature $(0,4)$, Conform. Geom. Dyn. 9 (2005) 46-75 MR2133805

[11] M G Katz, M Schaps, U Vishne, Logarithmic growth of systole of arithmetic Riemann surfaces along congruence subgroups, J. Differential Geom. 76 (2007) 399-422 MR2331526

[12] S Makisumi, A note on Riemann surfaces of large systole, J. Ramanujan Math. Soc. 28 (2013) 359-377 MR3113388

[13] H Parlier, Kissing numbers for surfaces, J. Topol. 6 (2013) 777-791 MR3100890

[14] H Parlier, Simple closed geodesics and the study of Teichmüller spaces, from: "Handbook of Teichmüller theory, IV”, (A Papadopoulos, editor), IRMA Lect. Math. Theor. Phys. 19, Eur. Math. Soc., Zürich (2014) 113-134 MR3289700

[15] B Petri, Random regular graphs and the systole of a random surface, preprint (2013) arXiv: $1311.5140 \mathrm{v} 1$

[16] P Przytycki, Arcs intersecting at most once, Geom. Funct. Anal. 25 (2015) 658-670 MR3334237

[17] P Schmutz, Congruence subgroups and maximal Riemann surfaces, J. Geom. Anal. 4 (1994) 207-218 MR1277506

[18] P Schmutz, Arithmetic groups and the length spectrum of Riemann surfaces, Duke Math. J. 84 (1996) 199-215 MR1394753

[19] P Schmutz Schaller, Extremal Riemann surfaces with a large number of systoles, from: "Extremal Riemann surfaces", (J R Quine, P Sarnak, editors), Contemp. Math. 201, Amer. Math. Soc. (1997) 9-19 MR1429190

Mathematics Institute, Zeeman Building

University of Warwick, Coventry CV4 7AL, UK

Department of Mathematics, University of Fribourg

Ch. du Musée 23, CH-1700 Fribourg, Switzerland

federica.fanoni@gmail.com, hugo.parlier@unifr.ch

Received: 1 September $2014 \quad$ Revised: 3 March 2015 
